\title{
Novel Lattice-Searching Method for Modeling the Optimal Strain-Free Close-Packed Isomers of Clusters
}

\author{
Longjiu Cheng and Jinlong Yang* \\ Hefei National Laboratory for Physics Sciences at Microscale, University of Science and Technology of China, \\ Hefei, Anhui, 230026, People's Republic of China
}

Received: September 28, 2006; In Final Form: January 24, 2007

\begin{abstract}
Small icosahedral, decahedral, and fcc structures have been studied by unbiased global optimization methods or Wulff construction and Northby lattice methods. Strain-free close-packed structures are not much discussed because the structures are very difficult to optimize and there is no common strain-free close-packed lattice. We propose a new strategy to construct such a lattice containing all possible strain-free close-packed isomers, and by searching the lattice with an efficient method the optimal close-packed structures were modeled. Testing with the Morse potential at $\rho_{0}=14.0$ for cluster size $10 \leq N \leq 250$ showed that optimal strain-free close-packed (scp) structures are lower in energy than fcc structures in most cases even for the well-known magic numbers of fcc structures (e.g., $N=201$ ). It was found that, due to the gaps in next-nearest-neighbor contacts, fcc will become energetically incomparable with scp at very large clusters with a pair potential. Moreover, compared with the results in the literature, some new global minima for Morse clusters at large $\rho_{0}$ values are given.
\end{abstract}

\section{Introduction}

Geometry optimization of clusters has attracted great interest by physical chemists. ${ }^{1}$ There are two kinds of methods for finding the low-energy motifs of clusters, i.e., unbiased methods and biased methods. Unbiased methods make use of a global optimization method to search the potential energy surface (PES) without any geometric knowledge. A number of efficient unbiased global optimization methods for cluster optimization have been developed. ${ }^{2-10}$ For large cluster sizes, unbiased methods may be very time-consuming, but they can locate unexpected motifs; e.g., the global minimum of Lennard-Jones (LJ) clusters at cluster size $N=98$ is a special tetrahedral packing, which was first located by an unbiased method. ${ }^{11}$

Biased methods utilize known geometry knowledge to make the problem easier. For small LJ clusters, icosahedra are optimal motifs, and then with cluster size increasing decahedra are optimal, and finally face-centered cubic (fcc) motifs are optimal. As shown in Figure 1, optimal fcc, icosahedral, and decahedral motifs with complete shells are truncated octahedron, Mackay icosahedron, and Marks decahedron, respectively, which can be given directly by the Wulff construction and its modifications. ${ }^{12-15}$

Construction methods can only give the motifs with certain complete outer shells. To optimize clusters with incomplete outer shells, Northby ${ }^{16}$ proposed a lattice-searching method and first located most of the icosahedral global minima of LJ clusters for cluster sizes $N \leq 150$. In the Northby lattice method, first a large and complete icosahedral lattice is built, and then by counting the nearest-neighbor contacts $(\mathrm{NN})$ it is quite easy to find the optimal icosahedral minima. With similar methods, Doye and Wales ${ }^{17}$ modeled optimal decahedral and fcc clusters up to $N=150$, Romero et al. ${ }^{18}$ located most of the known global minima of $\mathrm{LJ}_{150-309}$ including several decahedral ones, and more

* Corresponding author. Telephone: +86-551-3606408. Fax: +86-5513602969. E-mail: jlyang@ustc.edu.cn.
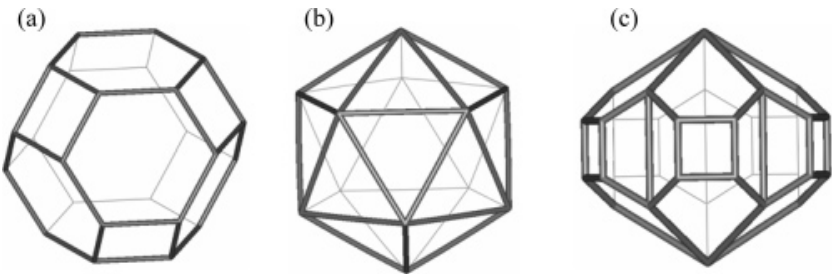

Figure 1. Structures of (a) truncated octahedron, (b) Mackay icosahedron, and (c) Marks decahedron.

recently, Xiang et al. ${ }^{19-21}$ located putative global minima of $\mathrm{LJ}_{310-1610}$, where both icosahedral and decahedral lattices were considered, and for icosahedral lattice the central vacancy was also investigated.

Icosahedra are predominant for small LJ clusters, and have been sufficiently studied. Mackay icosahedra grow by shells, so generally icosahedral isomers have the same inner core and only differ at the outer layers, which makes icosahedra easily optimized with the Northby lattice method. For the clusters with short-ranged pair interactions, e.g., short-ranged Morse clusters, ${ }^{22,23}$ icosahedra are too strained, so decahedral and strainfree close-packed (scp) clusters are predominant. Morse clusters can be taken as a test system with pair interaction:

$$
U_{\mathrm{M}}(r)=\epsilon \mathrm{e}^{\rho_{0}\left(1-r / r_{\mathrm{e}}\right)}\left[\mathrm{e}^{\rho_{0}\left(1-r / r_{\mathrm{e}}\right)}-2\right]
$$

where $\epsilon$ is the pair well depth, $r_{\mathrm{e}}$ is the equilibrium distance, and the parameter $\rho_{0}$ determines the potential range; larger $\rho_{0}$ means more short-ranged interaction. Decahedra can also be easily modeled by Wulff construction and the Northby lattice method, while scp clusters are still not so well studied.

Both fcc and hexagonal close-packed (hcp) lattices are strainfree. As shown in Figure 2, fcc lattice is packed by ABCABC... $\{111\}$ layers and hcp lattice is packed by $\mathrm{ABAB} . . .\{111\}$ layers, which can be easily modeled; e.g., complete regular truncated octahedral as shown in Figure 1a occur at $N=38,201,586$, 


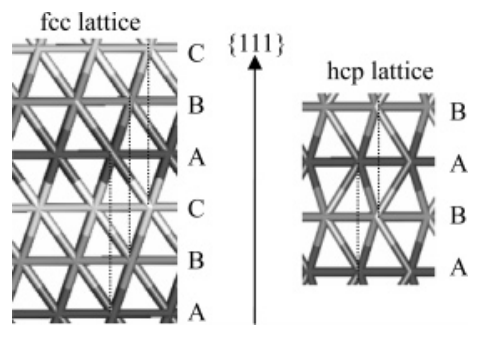

Figure 2. Two-dimensional projections of fcc and hep lattices. The labeled axis is the normal line of the $\{111\}$ layers. In fcc lattice there can be three kinds of $\{111\}$ layers labeled, "A", "B", and "C", so the fcc lattice can be thought as packed by $\mathrm{ABCABC}$... sequence. The sequence for the hcp lattice is $\mathrm{ABAB} . .$.

.... Figure 3 shows some typical scp motifs, which are known global minima of Morse clusters at $\rho_{0}=14.0 .^{23}$ It can be seen that scp motifs may contain various fcc and hep units, and can also be strain-free. Comparing different scp motifs shown in Figure 3, scp isomers may differ at both core and surface layers, so unlike fcc, icosahedral, and decahedral clusters as shown in Figure 1, there is no common scp lattice, and it is hard to optimize scp motifs directly using the traditional Northby lattice method. Moreover, for perturbation-based unbiased global optimization methods, scp motifs are notoriously difficult to optimize even at very small cluster size. ${ }^{24,25}$

In this work, we propose a new strategy to construct the scp lattice. Searching the constructed lattice by counting the nearestneighbor contacts (NN), new optimal scp isomers may be located. Testing with Morse clusters at $\rho_{0}=14.0$, even at magic numbers of the truncated octahedron, e.g., $N=201$, scp minima may be lower in potential energy.

\section{Methods}

2.1. Constructing the scp Lattice. The fcc lattice can be easily constructed. Figure 4 a shows a complete fcc octahedral lattice which has four directions of $\{111\}$ layers, and at each direction the layer sequence is $\mathrm{ABCABC}$.... As shown in Figure 3 , the scp lattice may contain fcc and hcp $\{111\}$ layer sequences at each direction. Although there is no simple lattice that contains all possible scp motifs, we can build such a lattice with superabundant sites. Actually, a similar strategy has been adopted by Manninen et al., ${ }^{26,27}$ where hcp layers are called "stacking faults", and all possible "stacking faults" were considered in their optimizations for locating the optimal closepacked structures with the hard sphere model. By turning each $\{111\}$ layer of Figure $4 a$ to $A+B+C$ as shown in Figure $4 b$, scp lattices with superabundant sites can be constructed as shown in Figure 4c, which may contain all possible $\{111\}$ layer sequences. In each $\{111\}$ direction of such a scp lattice, the added sites (white sites in Figure 4c) are twice as many as the original fcc sites (black sites in Figure 4c). There are four directions of $\{111\}$ layers, so the total added lattice sites are 8 times as many as the original fcc sites.

The scp lattice given in Figure $4 c$ is too large with too many superfluous sites and should be reduced. Figure 5a is a basic octahedron, which is the core of the lattice. It can be seen that the fcc lattice has four directions $(a, b, c$, and $d)$ of $\{111\}$ layer and three directions $(x, y$, and $z)$ of $\{100\}$ layer. The first reduction is to cut off the sites in the seven $\{111\}$ or $\{100\}$ layers leaving the origin larger than a parameter $R_{\text {cut }}$. If there is an hcp sequence in one $\{111\}$ direction, the other three directions of $\{111\}$ layer will be broken. Thus, the hep sequence can only appear in one direction of $\{111\}$ layer (as shown in Figure $3 a, c$ ) or in the surfaces (as shown in Figure $3 b$ ); i.e., there cannot be crossovers of hcp sequences at different $\{111\}$ directions. Based on the above regulation, the reduced scp lattice can be constructed as shown in Figure 5b. In direction $a$, all reasonable layer sequences are considered (Figure 5c), while in directions $b, c$, and $d$, only the surface hcp layers are considered (Figure 5d). In this way, the size of the lattice can be reduced; e.g., in the example of Figure 5, with $R_{\text {cut }}=3.2 r_{\mathrm{e}}$, sizes of the original fcc lattice, the reduced scp lattice, and the unreduced scp lattice are 260, 750, and 2267, respectively.

2.2. Searching the scp Lattice. Let $N_{\mathrm{L} 0}$ be the size of the fcc lattice (black sites in Figure $5 \mathrm{~b}$ ) and $N_{\mathrm{L}}$ be the size of the constructed scp lattice. Supposing the size of the cluster to be optimized is $N$, with a suitable $R_{\text {cut }}$ (in this work, $R_{\text {cut }}$ is chosen to make $N_{\mathrm{L} 0}=2 N-4 N$ ), each reasonable scp isomer may be a fragment of the lattice. Thus, optimization with such a lattice is a simple combinational optimization problem with searching space $C_{N_{\mathrm{L}}}^{N}$; i.e., choose $N$ suitable sites from the $N_{\mathrm{L}}$ lattice sites to compose an optimal solution. Here we evaluate a solution by counting the number of nearest neighbors (NN). We use $\theta$ $(r)$ to evaluate whether a pair of sites in the lattice is nearestneighbor. Simply, we set $\theta(r)=1$ when $r=r_{\mathrm{e}}$, and $\theta(r)=0$ when $r>r_{\mathrm{e}}$. Moreover, the scp lattice is superabundant, so there are many pairs of sites with distance $r<r_{\mathrm{e}}$, which is illegal for a reasonable structure, and so we assign it $\theta(r)=-10$.

In this work, we adopt a greedy strategy to search the lattice, which is somewhat similar to the lattice-searching strategy in ref 9. Details of the lattice-searching method are summarized as follows:

1. INITIALIZE: First, evaluate each pair of sites in the lattice $E_{\mathrm{L}}(i, j)=\theta\left(r_{i j}\right)\left(i, j=1,2, \ldots, N_{\mathrm{L}}\right)$. The greedy lattice-searching procedure will be repeated $N_{\text {try }}$ times. Initialize an empty bank with size $N_{\text {bk }}$ to record the best solutions in the $N_{\text {try }}$ times of the searching procedure. Set the iteration number $I=0$.

2. Randomly select $N$ sites from the lattice to generate a starting solution $S_{0}$, and calculate its $\mathrm{NN}$ with the established $E_{\mathrm{L}}(i, j)$. Then, calculate the NN of each site (occupied or not) in the lattice.

3 . With the current solution $S_{k}(k=0,1,2, \ldots)$, move the atom in the occupied site with the least NN to the vacant site with the most NN to form a new solution $S_{k}{ }^{\prime}$, and then update the NN of $S_{k}{ }^{\prime}$ and each site in the lattice simply by subtracting the contribution of the old site and adding the contribution of the new site. There may be more than one site with the least or the most NN; just choose one randomly.

4. If $S_{k}{ }^{\prime}$ has more NN than $S_{k}$, accept it as the starting solution of the next generation $\left(S_{k+1}\right)$ and return to step 3. Otherwise, take $S_{k}$ as the best solution of this single greedy lattice-searching procedure.

5. Calculate the potential energy of $S_{k}$. If there is no same solution with $S_{k}$ in the bank (the criterion is $\Delta E<1.0 e-7 \epsilon$ ) and the potential energy of $S_{k}$ is lower than that of the worst one in the bank, replace it. Increase the iteration number $I$ by 1 . If $I<N_{\text {try }}$, go back to step 2. Otherwise terminate the iteration.

6. FINALIZE: Minimize the potential energy of each solution recorded in the bank, and take the best solution as the putative optimal isomer contained in the lattice.

\section{Results and Discussion}

3.1. Numerical Performance. To investigate the performance of the lattice-searching method, Morse clusters at $\rho_{0}=14.0$ are chosen as a test case. For comparisons, optimal isomers of both scp and fcc motifs are optimized by searching scp and fcc lattices separately. The greedy lattice-searching procedure described above is extremely fast, but it may end at various 
(a)

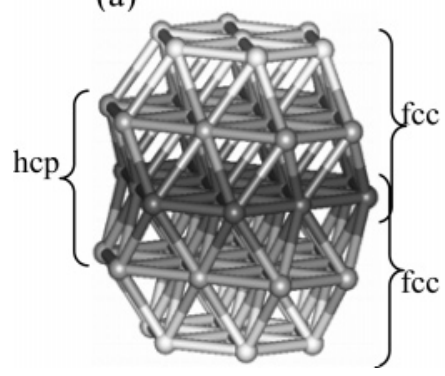

(b)

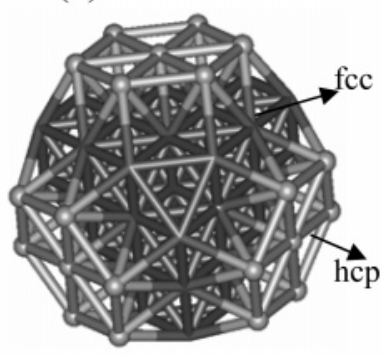

(c)

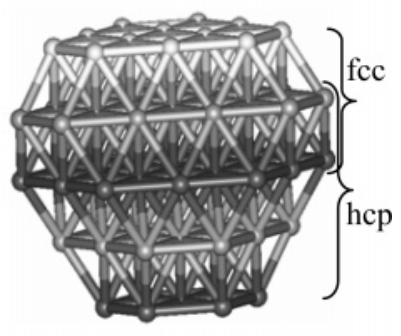

Figure 3. Illustrations of some selected optimal scp clusters: (a) $N=50$, the top and bottom three layers are fcc, while the middle three layers are hcp; (b) $N=59$, it has a truncated tetrahedral fcc core, and outside the four $\{111\}$ faces of the tetrahedral core there are four hcp layers; (c) $N=68$, the top three layers are fcc, while the bottom four layers are hcp.

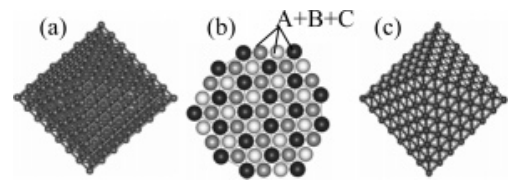

Figure 4. (a) Complete fcc octahedral lattice. (b) Congregation of the three kinds of $\{111\}$ layers $(A, B, C)$ at one layer. (c) Superabundant scp lattice by turning each $\{111\}$ layer of (a) to $A+B+C$ as $(b)$. The black sites are the same in (a), and the white sites are added potential hop sites.

minima. Thus, to ensure the optimization results, the greedy lattice-searching procedure is repeated for a very large number of runs ( $N_{\text {try }}=10^{6}$ in this study). Taking $M_{200}$ as an example, during the $10^{6}$ greedy lattice-searching runs, the optimal fcc and scp motifs are located for 7237 and 43 times, respectively, and the consumed time is about 5600 and 16000 s, respectively (both with a single Itanium2 $1.5 \mathrm{GHz}$ processor), which is acceptable.

3.2. Optimal fec and scp Isomers at $N=10-250$. With the newly developed method, putative optimal fcc and scp isomers of Morse clusters at $\rho_{0}=14.0$ for the size range $10 \leq$ $N \leq 250$ are located. ${ }^{28}$ The fcc lattice is a subaggregate of the scp lattice, so optimal scp isomers are not worse than relative optimal fcc isomers, and for some cases optimal scp isomers may be the same as the fcc ones.

For unstrained clusters with short-ranged interactions, $\mathrm{NN}$ is the most important factor determining the potential energy. Figure 6a compares the NN of optimal fcc and scp isomers. It can be seen that, for many cases, the NN of optimal scp isomers are larger than that of fcc isomers, and at some magic numbers (as labeled) the gap of $\mathrm{NN}$ is two. As a comparison, the results of ref 17 are also given in Figure 6a, where optimal fcc clusters at $10 \leq N \leq 150$ (may have hcp layers at surfaces) were modeled with the Northby lattice method. It can be found that, at small $N$, the results of ref 17 may be better than those of optimal fcc isomers due to the consideration of potential hcp layers at surfaces, but worse than those of optimal scp isomers for many cases, especially for larger $N$. Moreover, at $N=64$, $66,91,95,101,107$, and 109, the located optimal scp structures are larger in $\mathrm{NN}$ than that of ref 27 , where optimal structures with the hard sphere model up to $N=110$ were optimized with Monte Carlo simulations.

Besides NN, scp isomers with hep layers may have lower potential energy. Figure $6 \mathrm{~b}$ compared the potential energy of optimal fcc and scp isomers. It can be seen that except for very few cases $(N=38-40,132,136)$, optimal scp isomers have lower potential energy than fcc isomers even for the well-known magic number of truncated octahedron $(N=201)$. At the exception sizes, isomers with hcp layers cannot have sufficient large $\mathrm{NN}$ as fcc isomers. Moreover, testing for some other potentials, e.g., LJ potential and Girifalco pair potential ${ }^{29}$ for $\mathrm{C}_{60}$ molecular clusters, the potential energy of the optimal scp isomer is also lower than that of the optimal fcc isomer at $N=$ 201.

3.3. Most Stable Magic Numbers. Figure 7 plots $\mathrm{NN}-\mathrm{NN}_{\text {to }}$ of optimal fcc (Figure 7a) and scp (Figure 7b) isomers as a function of $N$, where $\mathrm{NN}_{\text {to }}$ is the interpolation for the optimal truncation octahedron (details can be found in ref 17). Positive peaks in the figure correspond to stable structures. It can be found that Figure $7 \mathrm{a}$ and Figure $7 \mathrm{~b}$ have similar outlines, but there are some new stable magic numbers for scp clusters and magic numbers for fcc clusters may disappear. Moreover, most of the worst cases in fcc clusters are improved in scp clusters.

Figure 8 plots the stable magic numbers in Figure 7 and some typical structures with special symmetry. The growth sequence of fcc structures (Figure 8a) can be very regular. By adding atoms around the eight $\{111\}$ faces or six $\{100\}$ faces, clusters grow gradually. The growth upon the $\{100\}$ faces can be more regular; e.g., the rather regular growth curve in Figure 7a at $N$ $=11-23$ and $105-152$ is mainly caused by the growth on the six $\{100\}$ faces. The stable structures have rather ordered $\{111\}$ and $\{100\}$ faces; e.g., $13 \mathrm{~F}, 38 \mathrm{~F}, 79 \mathrm{~F}, 116 \mathrm{~F}$, and $201 \mathrm{~F}$ are regular truncated octahedra with $O_{h}$ symmetry. By adding or truncating layers, other stable structures may be obtained: e.g., $52 \mathrm{~F}$ is $38 \mathrm{~F}$ plus a regular cap and $24 \mathrm{~F}$ is $38 \mathrm{~F}$ minus the cap; $86 \mathrm{~F}$ is $79 \mathrm{~F}$ plus a regular $\{111\}$ layer and $102 \mathrm{~F}$ is $79 \mathrm{~F}$ plus a regular cap; $182 \mathrm{~F}$ and $192 \mathrm{~F}$ are $201 \mathrm{~F}$ minus a $\{111\}$ and $\{100\}$ surface, respectively; $213 \mathrm{~F}$ and $244 \mathrm{~F}$ are $201 \mathrm{~F}$ plus a $\{111\}$ layer and a regular cap, respectively; $231 \mathrm{~F}$ is $213 \mathrm{~F}$ plus three $\{100\}$ layers.

The growth sequences of the scp structures (Figure $8 \mathrm{~b}$ ) are not so regular as fcc sequences due to its more growth pattern. $12 \mathrm{C}, 13 \mathrm{C}$, and $26 \mathrm{C}$ are hcp; 59C, 100C, 116C, and 180C are regular truncated tetrahedra plus four complete hcp surfaces, which have $T_{d}$ symmetry; $58 \mathrm{C}$ and $179 \mathrm{C}$ can be obtained by turning one of the hcp surfaces of $59 \mathrm{C}$ and $180 \mathrm{C}$ to fcc; $91 \mathrm{C}$ can be obtained by truncating $100 \mathrm{C} ; 189 \mathrm{C}$ is $180 \mathrm{C}$ plus a cap; $107 \mathrm{C}$ is $100 \mathrm{C}$ plus one fcc layer outer the hcp surface; $236 \mathrm{C}$ is 220C plus one hcp layer outer the hcp surface. The other structures in Figure $8 \mathrm{~b}$ may have various $\{111\}$ layer sequences of fcc and hcp at one direction.

For some cases of stable scp structures, e.g., $N=12,26,50$, $59,91,100,173,180,189,216$, and 238 , there is no relative stable fcc structure. The reason is that scp clusters may have a greater chance to form regular outer shells due to the admission of hcp layers. For some other cases, fcc and scp structures can both have regular outer shells: e.g., $116 \mathrm{C}$ can be translated to $116 \mathrm{~F}$ by turning the four hcp surfaces to fcc; $201 \mathrm{C}$ is packed by two $\{111\}$ face-sharing half parts of $201 \mathrm{~F}$. 

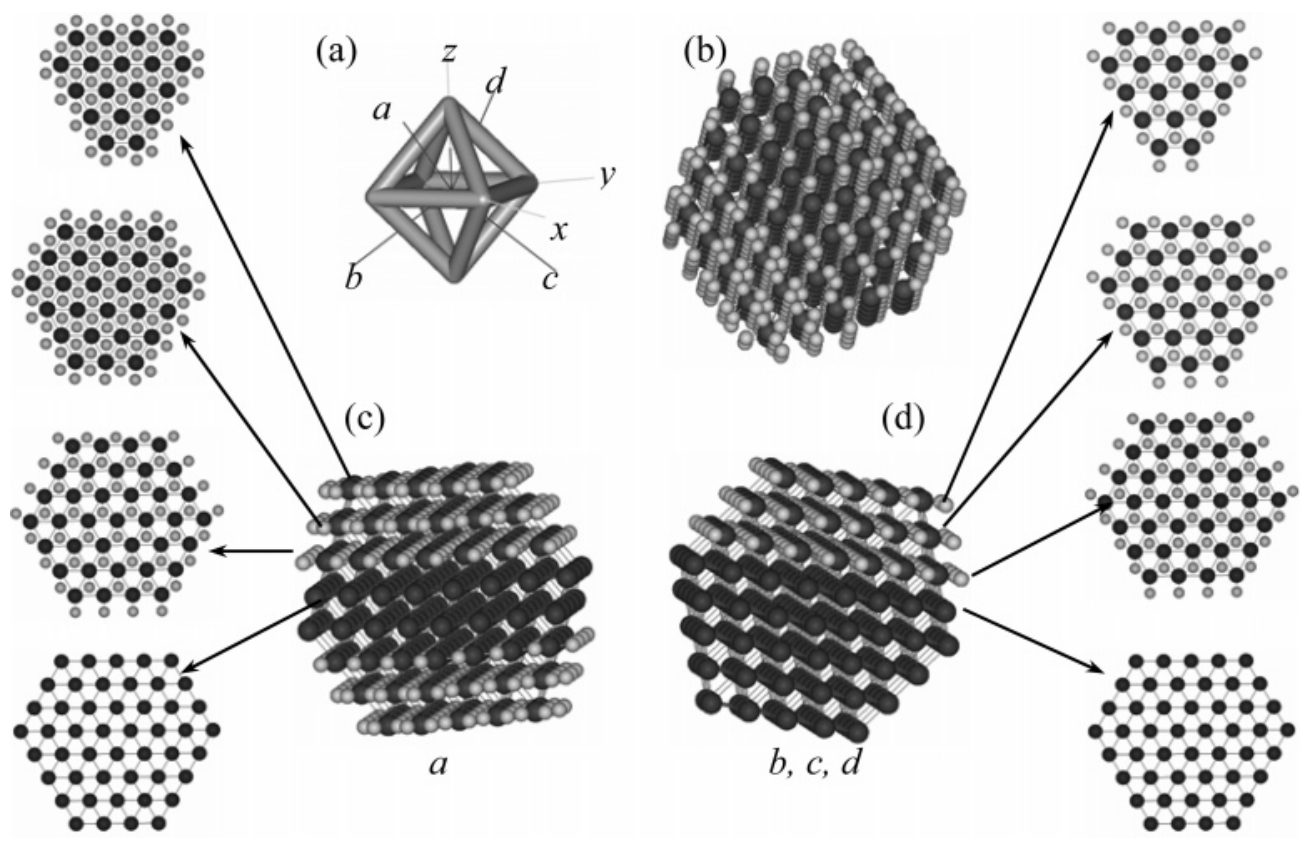

Figure 5. (a) Core of the octahedral lattice, which has four directions of the $\{111\}$ layer $(a, b, c$, and $d)$ and three directions of the $\{100\}$ layer $(x, y$, and $z$ ). (b) Reduced scp lattice, where the black sites are fcc lattice sites and the white sites are added potential hcp sites. (c) Layers in direction $a$ of (b), where the middle two layers are fixed and for the other layers all possible hcp layers are considered. As shown in the left of (c), the reduced layer sequence (from bottom to top) becomes $\mathrm{A}, \mathrm{B}+\mathrm{C}, \mathrm{A}+\mathrm{B}+\mathrm{C}, \mathrm{A}+\mathrm{B}+\mathrm{C}, \ldots$. (d) Layers in directions $b, c$, and $d$ of (b), where only surface hcp layers are considered. As shown in the right of (d), the reduced layer sequence (from bottom to top) is $\mathrm{A}, \mathrm{B}+\mathrm{C}, \mathrm{C}+\mathrm{A}, \mathrm{A}+$ $\mathrm{B}, \ldots$

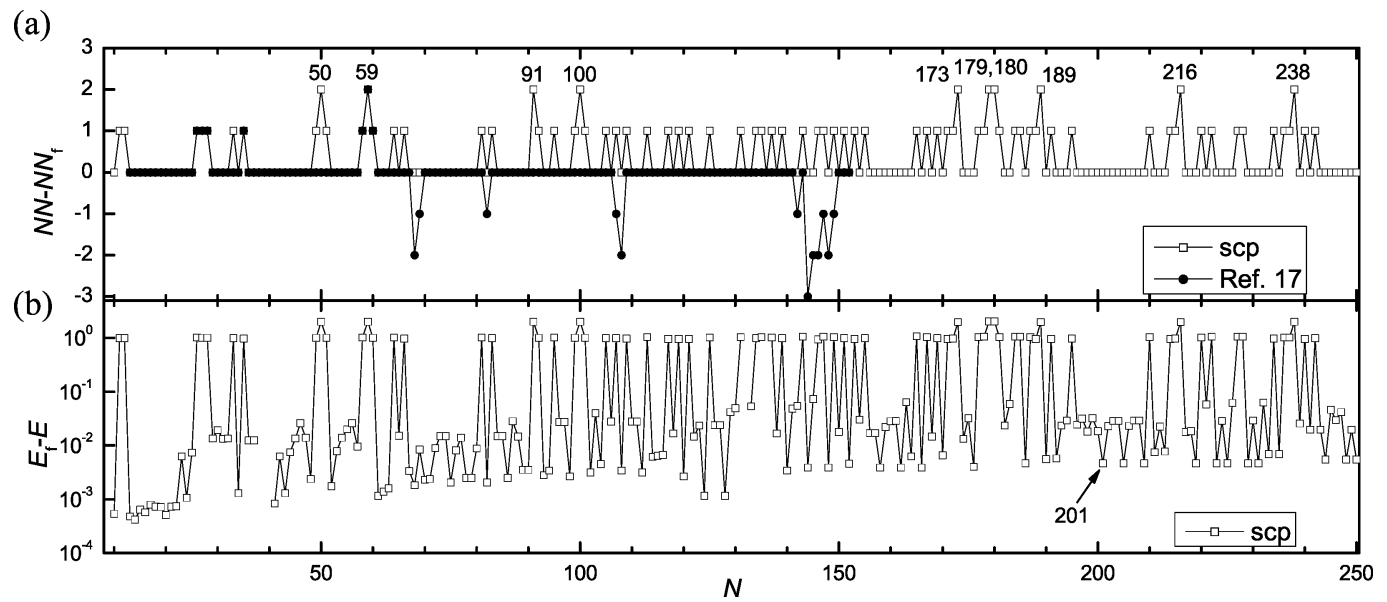

Figure 6. Plots of (a) $\mathrm{NN}-\mathrm{NN}_{\mathrm{f}}$ and (b) $E_{\mathrm{f}}-E$ as a function of $N$, where $\mathrm{NN}_{\mathrm{f}}$ and $E_{\mathrm{f}}$ are nearest-neighbor contacts and potential energy of the optimal fcc isomers, respectively.

3.4. Gaps of Next-NN. From fcc to scp structures, although the symmetry decreases and the NN may not increase, energy may decrease, which may be caused by the hcp units (or stacking faults) as studied in large LJ clusters. ${ }^{30-32}$ Moreover, for a potential with higher long-distance interaction the gaps are generally larger. For example, the energetic gaps between 116C and 116F for Morse potential at $\rho_{0}=14.0$, Girifalco potential, and LJ potential are $-0.006687,-0.136695$, and -0.590505 (the unit is $\epsilon$ ), respectively, and for $N=201$, the gaps are $-0.004642,-0.150854$, and -0.760477 , respectively.

$13 \mathrm{~F}$ and $13 \mathrm{C}$ (as shown in Figure 8) have same NN, and $13 \mathrm{C}$ is the simplest case with a stacking fault. To investigate how stacking faults affect the potential energy, Table 1 gives the pair distance function (PDF) of $13 \mathrm{~F}$ and $13 \mathrm{C}$. The energy gap with the LJ pair potential at each pair distance is also given in the table. It can be seen that $13 \mathrm{C}$ and $13 \mathrm{~F}$ have the same $\mathrm{NN}$ and second-NN, but the third-NN in $13 \mathrm{C}$ is caused by the stacking faults. Therefore, stacking faults can make scp structures have higher next-NN and so have lower potential energy.

To investigate the effects of next-NN with the relationship of the potential range, Figure 9 plots the gaps of average binding energy per atom $\left(E_{\mathrm{bd}}\right)$ between fcc and scp structures with the function of $\rho_{0}$ at the magic numbers of $N=13,68,116$, and 201, where scp and fcc have same NN. It can be seen that, at $N=13,116$, and 201, $E_{\mathrm{bd}}$ of scp is higher than that of fcc at all potential ranges. Moreover, the gaps of $E_{\mathrm{bd}}$ increase with increasing potential range (with $\rho_{0}$ decreasing), which indicates that the gaps of next-NN can be enlarged by a higher long distance interaction. It is much different for $N=68$, where $68 \mathrm{C}$ is even worse than $68 \mathrm{~F}$ at very small $\rho_{0}$, which may be because $68 \mathrm{C}$ is much less spherical than $68 \mathrm{~F}$ and so its energy of non$\mathrm{NN}$ is smaller than $68 \mathrm{~F}$. However, with $\rho_{0}$ increasing, the energy of non-NN decreases more rapidly than that of next-NN, and so $68 \mathrm{C}$ is better than $68 \mathrm{~F}$ at large $\rho_{0}$. Moreover, the number of 
(a)

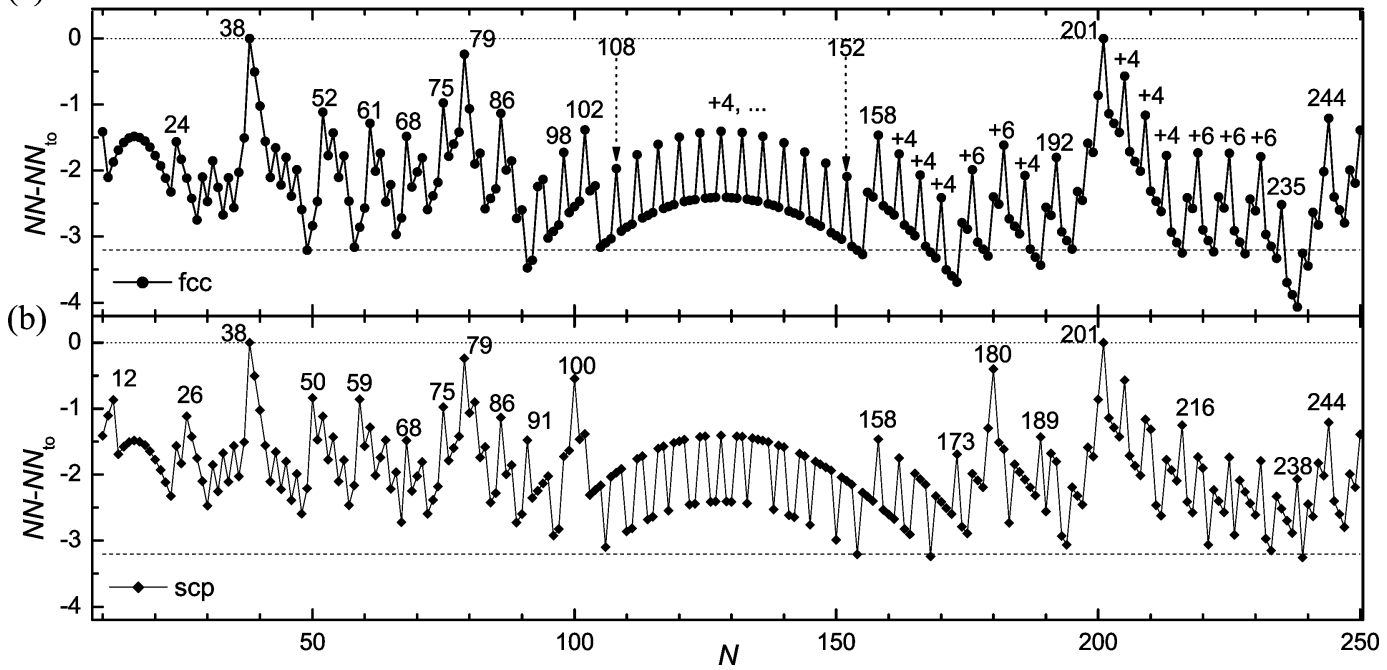

Figure 7. Plots of $\mathrm{NN}-\mathrm{NN}_{\text {to }}$ of (a) optimal fcc isomers and (b) optimal scp isomers as a function of $N$. The dotted line is the interpolation for the truncation octahedron with regular hexagonal faces (as shown in Figure 1a), and the dashed line is a reference line at $\mathrm{NN}-\mathrm{NN}_{\mathrm{to}}=-3.2$.

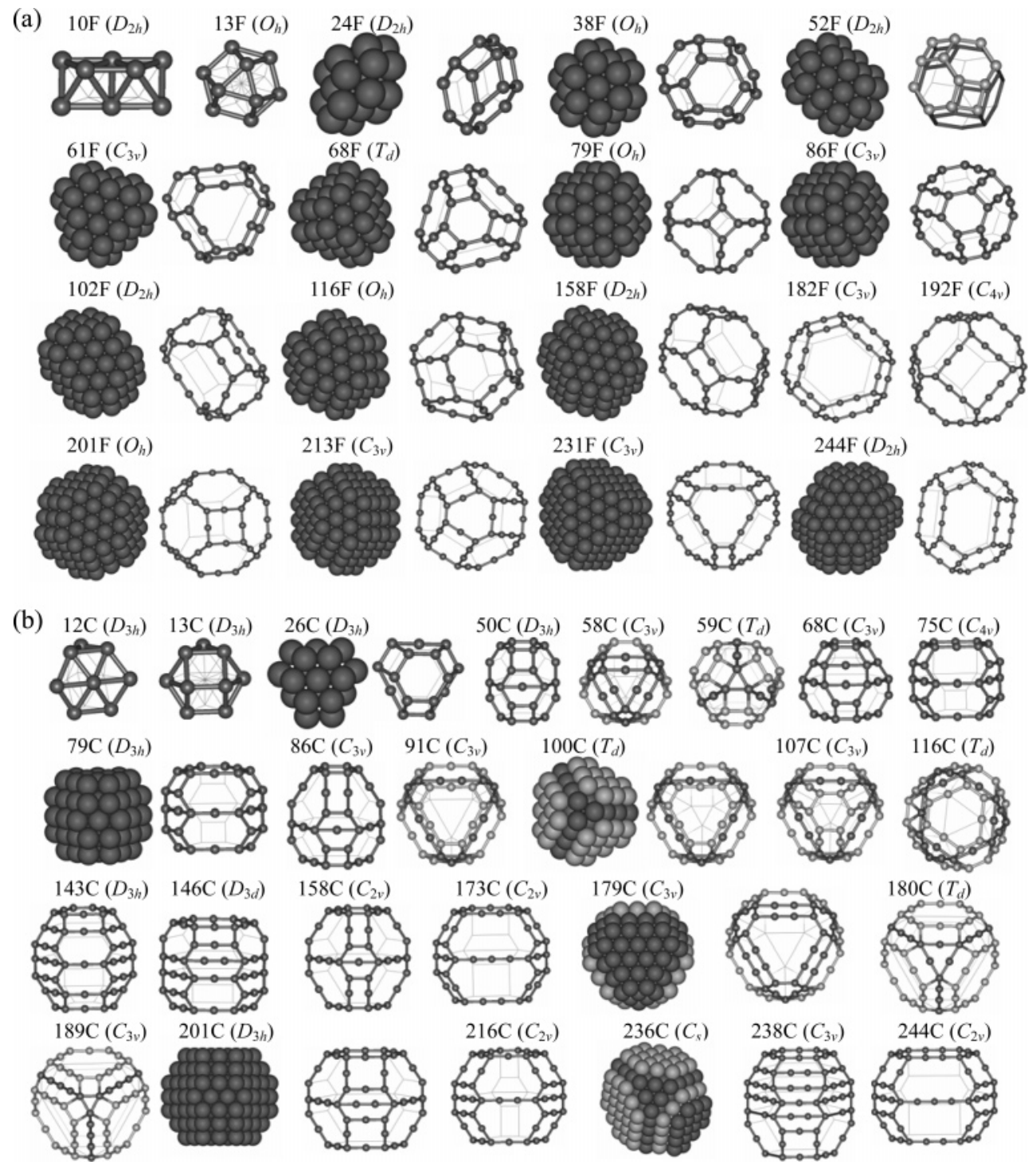

Figure 8. Most stable or typical (a) fcc structures and (b) scp structures. The structures are given in either the space-filling model or the polyhedron model with only atoms and bonds on the edges shown. The surface hcp layers are shown in light gray. Cluster sizes and point groups are labeled.

hcp units (stacking faults) and the number of $\{111\}$ layers in $13 \mathrm{C}, 68 \mathrm{C}, 116 \mathrm{C}$, and $201 \mathrm{C}$ are $1 / 3,2 / 5,4 / 6$, and $1 / 7$, respectively, which correspond to the relative amount of the gaps of $E_{\mathrm{bd}}$ at large $\rho_{0}$ of the selected cases. 
TABLE 1: Pair Distance Function (PDF) of $13 \mathrm{~F}$ and $13 \mathrm{C}^{a}$

\begin{tabular}{lcrrr}
\hline & & \multicolumn{2}{c}{ number } & \\
\cline { 3 - 4 } index & pair distance $\left(r_{\mathrm{e}}\right)$ & $13 \mathrm{~F}$ & $13 \mathrm{C}$ & $E_{\mathrm{f}}-E_{\mathrm{c}}(\epsilon)$ \\
\hline 1 & 1.000 & 36 & 36 & 0.000 \\
2 & 1.414 & 12 & 12 & 0.000 \\
3 & 1.633 & 0 & 3 & 0.308 \\
4 & 1.732 & 24 & 18 & -0.436 \\
5 & 1.915 & 0 & 6 & 0.241 \\
6 & 2.000 & 6 & 3 & -0.093 \\
$>$ total $^{b}$ & & 78 & 78 & 0.020
\end{tabular}

${ }^{a} E_{\mathrm{f}}-E_{\mathrm{c}}$ gives the energy gaps of $13 \mathrm{~F}$ and $13 \mathrm{C}$ in the pair distance with the LJ pair potential. The unit of the distance is the equilibrium $r_{\mathrm{e}}$, and the unit of potential energy is the pair well depth $\epsilon .{ }^{b}$ For an easy comparison, the structures are not relaxed, and after relaxation with $\mathrm{LJ}$ potential the gap is $0.037 \epsilon$.

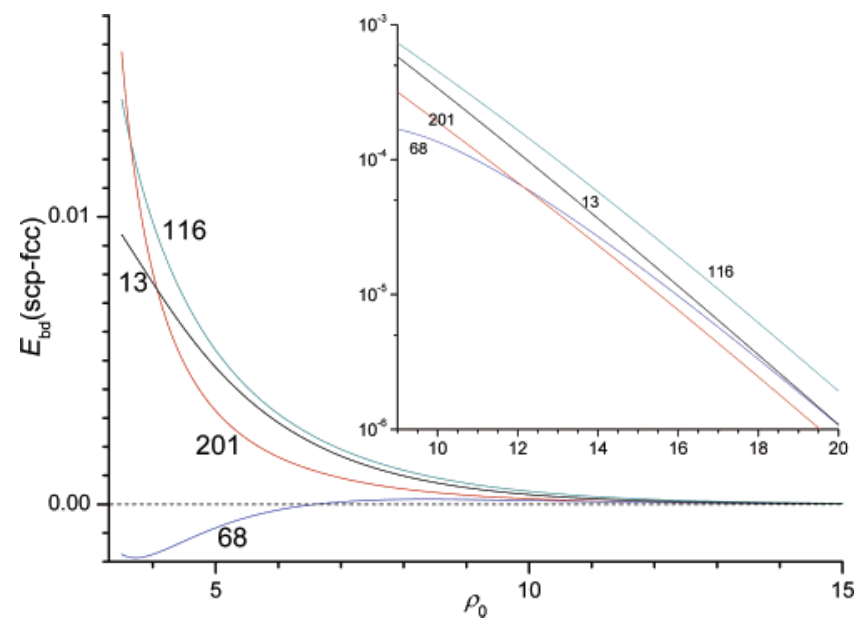

Figure 9. Gaps of $E_{\mathrm{bd}}$ with the Morse pair potential between optimal fcc and scp structures as a function of potential range $\rho_{0}$ at cluster sizes $N=13,68,116$, and 201 (as labeled in the figure). A positive value means scp has lower potential energy. The inset is an enlargement of the area at large $\rho_{0}$.

Many of the putative global minima of $\left(\mathrm{C}_{60}\right)_{N}$ clusters for $N$ $\leq 150$ are scp, ${ }^{33}$ and one can expect that for slightly larger $N$ scp clusters will be predominant. The well-known growth sequence with cluster size for LJ clusters is from icosahedra to decahedra, and finally to fcc. Mackay icosahedron is packed by 20 vertex-sharing distorted fcc tetrahedra, and for each tetrahedron, its three $\{111\}$ faces are shared with others, so there are $30(20 \cdot 3 / 2)$ hcp units. This makes icosahedra have not only more NN but also more next-NN. Similarly, there are five hcp units in the decahedron.

For each kind of ordered motif, the NN of the inner atoms is 12 , so the difference of NN only occurs at the surfaces, while the gaps of next-NN can occur at the interior. With cluster size increasing, the ratio of surface atoms decreases, so the effects of next-NN become more and more important compared to NN. Therefore, agreeing with Raoult et al., ${ }^{31}$ with cluster size increasing, energy gaps caused by next-NN between fcc and scp may become larger and larger. Thus, for large LJ clusters, global minima should be scp instead of fcc, and the transition size from decahedra to strain-free structures should decrease.

It should be pointed out that the role of next-NN discussed in this section is only for the pair potentials, and may be much different for an $n$-body potential. But even for a potential disfavoring the stacking faults, scp structures should also be considered at not too large cluster size instead of only fcc, because scp may have more NN at some cases (as shown in Figure 6a).
3.5. New Global Minima of Morse Clusters at Large $\rho_{0}$. Putative global minima of Morse clusters with $\rho_{0}$ for $N \leq 80$ and $N=147$ can be found in $\mathrm{CCD},{ }^{23}$ which were first reported by Doye et al. ${ }^{34,35}$ and have been subsequently updated by the same authors and others. At very small $\rho_{0}$, disordered structures are predominant. However, with $\rho_{0}$ increasing, icosahedral, decahedral, and scp structures become predominant in turn. For $\rho_{0}=14.0$, at cluster size $N=38-40,51-53,59-61,68,79$, and 80 , the global minima are scp; at $N=10,11$, and 13 , the global minima are icosahedral; at the other cluster sizes the global minima are decahedral. At very large $\rho_{0}$, global minima of Morse clusters should be strain-free; i.e., the optimal scp structures will be global minima. By comparison, we found some new global minima for very large $\rho_{0}$. For $N=10,13-22$, putative global minima listed in CCD for very large $\rho_{0}$ are decahedral, which are strained and cannot be optimal for an extremely short-ranged pair potential. For $N=23,64-67,76$, 77 , and 147, putative global minima given in CCD are scp but not optimal.

\section{Conclusions}

In conclusion, we developed an efficient method for modeling the scp structures, which have been less studied in literature compared to fcc or hcp ones. With consideration of all possible hcp and fcc layer sequences, a lattice with superabundant sites can be constructed, and all possible scp isomers can be contained in the lattice. There are no crossovers for two hcp layer sequences, so the scp lattice can be greatly reduced. We developed an efficient lattice-searching method to find optimal structures. Taking Morse clusters at $\rho_{0}=14.0$ as a test case, optimal scp structures are better than fcc structures at most cases of $10 \leq N \leq 250$ even for the well-known magic numbers of fcc structures (e.g., $N=201$ ). Moreover, some new scp global minima for Morse clusters at large $\rho_{0}$ are located. For a pair potential, the energy of fcc will become incomparable with scp at very large cluster size due to the gaps of next-NN. Therefore, for very large LJ clusters, scp structures will be predominant in potential energy instead of fcc structures, and the transition size from decahedral to close packed should decrease. For some clusters with short-ranged potential, e.g., $\mathrm{C}_{60}$ molecular clusters or silver clusters, scp structures can be expected to be predominant at not-so-large cluster size.

Acknowledgment. This work is partially supported by the National Natural Science Foundation of China (50121202, 20533030, 10474087), by the USTC-HP HPC project, and by the SCCAS and Shanghai Supercomputing Center.

Supporting Information Available: Zip file of structures in $x y z$ format. This material is available free of charge via the Internet at http://pubs.acs.org.

\section{References and Notes}

(1) Baletto, F.; Ferrando, R. Rev. Mod. Phys. 2005, 77, 371

(2) Deaven, D. M.; Ho, K. M. Phys. Rev. Lett. 1995, 75, 228.

(3) Wales, D. J.; Doye, J. P. K. J. Phys. Chem. A 1997, 101, 5111.

(4) Wales, D. J.; Scheraga, H. A. Science 1999, 285, 1368.

(5) Hartke, B. J. Comput. Chem. 1999, 20, 1752.

(6) Johnston, R. L. J. Chem. Soc., Dalton Trans. 2003, 22, 4193.

(7) Lee, J.; Lee, I. H.; Lee, J. Phys. Rev. Lett. 2003, 91, 080201. 309.

(8) Cheng, L. J.; Cai, W. S.; Shao, X. G. Chem. Phys. Lett. 2004, 389,

(9) Shao, X. G.; Cheng, L. J.; Cai, W. S. J. Comput. Chem. 2004, 25, 1693.

(10) Pullan, W. J. Comput. Chem. 2005, 26, 899.

(11) Leary, R. H.; Doye, J. P. K. Phys. Rev. E 1999, 60, R6320.

(12) Wulff, G. Z. Kristallogr. 1901, 34, 449. 
(13) Mackay, A. L. Acta Crystallogr. 1962, 15, 916.

(14) Marks, L. D. J. Cryst. Growth 1983, 61, 556.

(15) Cleveland, C. L.; Landman, U. J. Chem. Phys. 1991, 94, 7376.

(16) Northby, J. A. J. Chem. Phys. 1987, 87, 6166.

(17) Doye, J. P. K.; Wales, D. J. Chem. Phys. Lett. 1995, 247, 339.

(18) Romero, D.; Barrón, C.; Gómez, S. Comput. Phys. Commun. 1999, 123,87 .

(19) Xiang, Y. H.; Jiang, H. Y.; Cai, W. S.; Shao, X. G. J. Phys. Chem. A 2004, 108, 3586 .

(20) Xiang, Y. H.; Cheng, L. J.; Cai, W. S.; Shao, X. G. J. Phys. Chem. A 2004, 108, 9516

(21) Shao, X. G.; Xiang, Y. H.; Cai, W. S. J. Phys. Chem. A 2005, 109, 5193.

(22) Morse, P. M. Phys. Rev. 1929, 34, 57.

(23) Wales, D. J.; Doye, J. P. K.; Dullweber, A.; Hodges, M. P.; Naumkin, F. Y.; Calvo, F.; Hernández-Rojas, J.; Middleton, T. F. The Cambridge Cluster Database (CCD). http://www-wales.ch.cam.ac.uk/ CCD.html.

(24) Roberts, C.; Johnston, R. L.; Wilson, N. T. J. Chem. Soc., Theor Chem. Acc. 2000, 104, 123.
(25) Doye, J. P. K.; Leary, R. H.; Locatelli, M.; Schoen, F. INFORMS J. Comput. 2004, 16, 371.

(26) Manninen, K.; Manninen, M. Eur. Phys. J. D 2002, 20, 343.

(27) Manninen, K.; Akola, J.; Manninen, M. Phys. Rev. B 2003, 68 235412 .

(28) Coordinates of the optimal fcc and close-packed structures will be available online from the CCD. ${ }^{23}$ The point group, nearest-neighbor contacts $(\mathrm{NN})$, and potential energy of Morse potential at $\rho_{0}=14.0$ of each structure are also listed there.

(29) Girifalco, L. A. J. Phys. Chem. 1992, 96, 858.

(30) Kihara, T.; Koba, S. J. Phys. Soc. Jpn. 1952, 7, 348

(31) Raoult, B.; Farges, J.; De Feraudy, M. F.; Torchet, G. Philos. Mag. $B$ 1989, 60, 881 .

(32) van de Waal, B. W. J. Chem. Phys. 1993, 98, 4909.

(33) Cheng, L. J.; Cai, W. S.; Shao, X. G. ChemPhysChem. 2005, 6 , 261

(34) Doye, J. P. K.; Wales, D. J.; Berry, R. S. J. Chem. Phys. 1995, $103,4234$.

(35) Doye, J. P. K.; Wales, D. J. J. Chem. Soc., Faraday Trans. 1997, 93, 4233. 